\title{
Co-Primary Spectrum Sharing with Resource Allocation in Small Cell Network
}

\author{
Tachporn Sanguanpuak* ${ }^{* \dagger}$, Nandana Rajatheva ${ }^{\dagger}$, Matti Latva-Aho ${ }^{\dagger}$ \\ *Department of Telecommunication Communication Engineering, Asian Institute of Technology, Thailand, \\ ${ }^{\dagger}$ Department of Communication Engineering, University of Oulu, P.O.Box 4500, FI-90014, Finland,
}

\begin{abstract}
We study co-primary spectrum sharing concept in small cell multiuser networks. Downlink transmission is considered with Rayleigh fading in interfering broadcast channel. Multiple-input-single-output (MISO) system is considered in two small cells. The sum rate maximization problem is studied in two cells having multiple users with multiple subcarriers. Zeroforcing (ZF) precoders are considered at both base stations. The problem becomes non-convex then and we factor the main objective problem into two subproblems. First subproblem is multiuser with subcarriers allocation where we assume that each subcarrier can be allocated to multiple users. Gale-Shapley algorithm is proposed for subcarrier allocation problem. Second subproblem is where ZF precoders are employed at both base stations for allocating power. It leads to water-filling based power allocation for both base stations.
\end{abstract}

\section{INTRODUCTION}

Nowadays, spectrum sharing concept is an important aspect to improve spectral efficiency. Authorized shared access (ASA) or licensed shared access (LSA) is a new concept that allows license holders (or incumbent users) to share spectrum with other service providers, under suitable conditions. Coprimary spectrum sharing is another concept which is designed to enable sharing spectrum between primary users.

In [1], LSA concept in $2.3 \mathrm{GHz}$ spectrum band is demonstrated for spectrum sharing between mobile network operator and incumbent users. The smart antennas technologies is used to enhance LSA systems [2]. The implementation scenarios of LSA by using active antenna is proposed to reduce spectrum exclusion and interference detection. Different types of incumbent users and factors for allowing spectrum sharing with LSA from their perspective are considered in [3]. In [4], the authors consider co-primary spectrum sharing for dense small cells. Spectrum efficiency is improved by making small cells utilize available spectrum efficiently through intra operator spectrum allocation and inter operator spectrum management. Simulations show the verification of spectrum efficiency improvement.

The spectrum sharing in cognitive radio aspect is studied in [5]. Unlicensed secondary users maximize their capacity by cooperating with primary users. The primary user will choose a secondary user as a relay. Then, based on relay selection in secondary users, power splitting, and transmit power, maximization the secondary capacity are explored. Maximization the minimum transmission capacity link of the secondary user with guaranteeing the target capacity of the primary pair is studied.
Sharing spectrum between the co-located radio networks which supported by different operators is studied in [6]. The spectrum is partitioned into non-orthogonally shared frequency sub-bands to maximize the inter radio access (inter-RAN) networks. A zero-forcing pre-coder is used in each dedicated frequency sub-bands while a sparse pre-coder is used to serve the scheduled users in shared frequency sub-bands. With user grouping, spectrum partitioning and user scheduling optimized, the maximum sum rate optimization is studied.

Stackelberg game formulation is proposed in two-tier femtocell network which contains one macro base station (MBS) and many spectrum sharing femtocells in [7]. MBS acts as a leader and femto base stations (FBS) act as followers. The MBS adjusts it power and imposes interference price on femtocells to maintain its users minimum rate requirement and earns revenue. Then, FBSs optimize power based on the imposed interference price which takes the cost of both spectrum sharing and energy usage into account.

In [8], the achievable rate region that can be achieved by using beamforming vectors that satisfy the power constraint is defined. Moreover, the Pareto boundary for achievable rate region for interference channel is provided. They propose an optimization method to compute the Pareto boundary of the achievable rate region for the two-user multi-input singleoutput (MISO) interference channel.

In our work, we propose spectrum sharing in multiuser two small cells network. The small cell base stations employ multiple antennas, each user is equipeed with a single antenna. Zero-forcing (ZF) precoder and subcarriers allocation for downlink transmission are considered in our optimization problem. We assume that both base stations allocate users in dedicated subbands and shared subbands. Each base station allocates it's users to utilize the shared bands when the number of subcarriers is not enough to serve overall users.

The sum rate maximization of multiple users with multiple subcarriers allocation problem is proposed. The problem becomes non-convex and therefore we separate the main problem into two optimization subproblems. In the first subproblem, we allocate subcarriers to users by using Gale-Shapley algorithm [9] by assuming that each particular subcarrier can be allocated to multiple users. The number of users which are served by each subcarrier is less than or equal to number of transmitting antennas. In the second subproblem, ZF precoder is employed. Thus, both stations allocate power by water-filling based on ZF precoder. 


\section{System Model}

Both BSs allocate users in their dedicated bands first and then allocate in the shared bands. In each cell, the base station allocates same amount of bandwidth to each set of users.

The base stations employ multiple number of antennas such that $N_{T_{k}}, N_{T_{j}}$ for base station $k$, and $j$, where $j \neq k$ respectively. At base station $k$, the number of total users is more than the number of transmit antennas denoted as, $I_{k} \geq N_{T_{k}}$, where $I_{k}$ is number of users in dedicated band of the cell $k$.

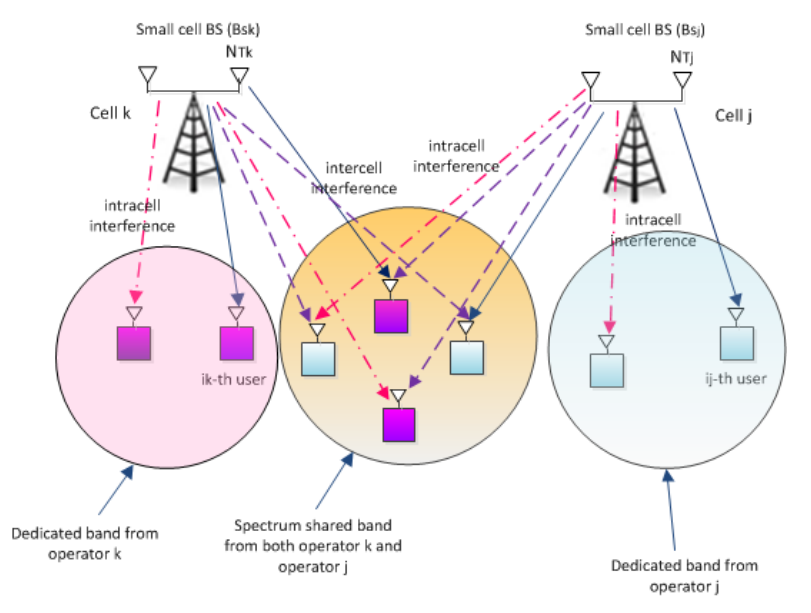

Fig. 1. Spectrum Sharing Between Two Small Cells

To allow simultaneous transmission to multiple users on the same subcarrier $n$, with the beamforming vector at the transmitter, we can formulate as

$$
\mathbf{y}_{n}=\mathbf{H}_{n} \mathbf{T}_{n} \mathbf{x}_{n}+\mathbf{z}_{n},
$$

where $\mathbf{T}_{n}=\left[\mathbf{T}_{1, n}, \ldots, \mathbf{T}_{I_{k}, n}\right]$ is the $N_{T_{k}} \times I_{k}$ transmit beamforming matrix where each component $\mathbf{T}_{i_{k}, n}=$ $\left[T_{i_{k}, n}^{1}, \ldots, T_{i_{k}, n}^{N_{T_{k}}}\right]^{T}$ is the $N_{T_{k}} \times 1$ beamforming vector allocated to user $i_{k}$ on subcarrier $n$ and $\mathbf{x}_{n}=\left[x_{1, n}, \ldots, x_{\mathcal{I}_{k}, n}\right]^{T}$ is the $\mathcal{I}_{k} \times 1$ column vector representing transmitted symbols to different $I_{k}$ users. $\mathbf{H}_{n}=\left[\mathbf{H}_{1, n}^{T}, \ldots, \mathbf{H}_{\mathcal{I}_{k}, n}^{T}\right]^{T}$ is the $I_{k} \times N_{T_{k}}$ complex channel gain matrix on subcarrier $n$ and $\mathbf{H}_{i_{k}, n}=$ $\left[H_{i_{k}, n}^{1}, \ldots, H_{i_{k}, n}^{N_{T_{k}}}\right]^{T}$ is the channel gain between BS and any user $i_{k} \in I_{k}$ in the cell $k$ on subcarrier $n . z_{i_{k}, n}$ is additive white Gaussian noise with zero mean and covariance matrix $\sigma_{i_{k}, n}^{2} \cdot \mathbf{y}_{n}=\left[y_{1, n}, \ldots, y_{\mathcal{I}_{k}, n}\right]^{T}$ is the $\mathcal{I}_{k} \times 1$ received vector whose components are the received signal by different users $\left\{1, \ldots, \mathcal{I}_{k}\right\}$, respectively.

Thus, in the dedicated band of the cell $k$, the received signal by user $i_{k}$ on subcarrier $n$ is given by,

$$
y_{i_{k}, n}=\mathbf{H}_{i_{k}, n} \mathbf{T}_{i_{k}, n} s_{i_{k}, n}+\sum_{j=1, j \neq i_{k}}^{\mathcal{I}_{k}} \mathbf{H}_{i_{k}, n} \mathbf{T}_{j_{k}, n} s_{j_{k}, n}+z_{i_{k}, n},
$$

where $\sum_{j=1, j \neq i}^{\mathcal{I}_{k}} \mathbf{H}_{i_{k}, n} \mathbf{T}_{j_{k}, n} s_{j_{k}, n}$ is the intracell interference (ICI). It is required to select $t_{k} \leq N_{T_{k}}$ users out of $\mathcal{I}_{k}$ users in each subcarrier. Number of simultaneously served users on each subcarrier is limited by the number of transmit antennas. There are $C_{k}=\sum_{t_{k}=1}^{N_{T_{k}}}\left(\begin{array}{c}\mathcal{I}_{k} \\ t_{k}\end{array}\right)$ combinations of users who can utilize same subcarrier, each of them is denoted as $\mathcal{A}_{t_{k}}$, where $\mathcal{A}_{t_{k}}=\left\{1, \ldots, \mathcal{I}_{k}\right\}, 0<\left|\mathcal{A}_{t_{k}}\right| \leq N_{T_{k}}$ denotes the cardinality of set $\mathcal{A}_{t_{k}}$. Assuming that the group of users $\mathcal{A}_{t_{k}}$ is assigned to subcarrier $n$, then received signal by any user $\left(i_{k} \in \mathcal{A}_{t_{k}}\right)$ is as, $y_{i_{k}, n}=\mathbf{H}_{i_{k}, n} \mathbf{T}_{i_{k}, n} s_{i_{k}, n}+\sum_{j_{k} \in \mathcal{A}_{t_{k}}, j_{k} \neq i_{k}} \mathbf{H}_{i_{k}, n} \mathbf{T}_{j_{k}, n} s_{j_{k}, n}+$ $z_{i_{k}, n}$, where $\sum_{j_{k} \in \mathcal{A}_{t_{k}}, j \neq i_{k}} \mathbf{H}_{i_{k}, n} \mathbf{T}_{j_{k}, n} s_{j_{k}, n}$ denotes the interference caused by users on the same subcarrier $n$. The total achievable rate at user $i_{k}$ in the cell $k$ is given by,

$$
R_{i_{k}}=\sum_{n=1}^{N} \sum_{t_{k}=1}^{C_{k}} \rho_{i_{k}, n, t_{k}} \log _{2}\left(1+\Gamma_{i_{k}, n}\right)
$$

where $\Gamma_{i_{k}, n}=\frac{\left|\mathbf{H}_{i_{k}, n} \mathbf{T}_{i_{k}, n}\right|^{2}}{\left|\sum_{j_{k} \in \mathcal{A}_{t_{k}}, j_{k} \neq i_{k}} \mathbf{H}_{i_{k}, n} \mathbf{T}_{j_{k}, n} s_{j_{k}, n}+z_{i_{k}, n}\right|^{2}}$. In the cell $j$, we can express the total achievable rate $R_{i_{j}}$ at user $i_{j}$ in the cell $j$ similar to (3). We consider to maximize the total rate of two cells in both dedicated band and shared band part. We assume that the number of subcarriers in dedicated band of cell $k$ and $j$ where $k \neq j$ is $\mathcal{N}$ and $\mathcal{M}$, respectively.

In the shared band part, both intracell and intercell interference affect at all users. The intracell interference is caused by the same subcarrier allocated to users and the intercell interference is caused by the broadcast signal from the other base station in the same frequency for shared band.

Thus, we can formulate the weighted sum rate maximization problem as,

$$
\max \quad \sum_{i_{k} \in I_{k}} R_{i_{k}}+\sum_{i_{j} \in I_{j}} R_{i_{j}}
$$

subject to $\quad \sum_{i_{k} \in I_{k}} \sum_{n \in \mathcal{N}}\left\|\mathbf{T}_{i_{k}, n}\right\|^{2} \leq P_{k}^{\max }$,

$$
\begin{gathered}
\sum_{i_{j} \in \mathcal{I}_{j}} \sum_{m \in \mathcal{M}}\left\|\mathbf{T}_{i_{j}, m}\right\|^{2} \leq P_{j}^{\max } \\
\sum_{i_{k} \in \mathcal{I}_{k}} \rho_{i_{k}, n, t_{k}} \leq N_{T_{k}} \quad \forall n, t_{k} \\
\sum_{i_{j} \in \mathcal{I}_{j}} \rho_{i_{j}, m, t_{j}} \leq N_{T_{j}} \quad \forall m, t_{j} \\
\rho_{i_{k}, n, t_{k}}, \rho_{i_{j}, m, t_{j}} \in\{0,1\} \quad \forall n, m, t_{k}, t_{j}
\end{gathered}
$$

where $\rho_{i_{k}, n, t_{k}}$ denotes whether subcarrier $n$ is assigned to user $i_{k} \in \mathcal{A}_{t_{k}}$ in the cell $k$, then $\rho_{i_{k}, n, t_{k}}=1$, otherwise $\rho_{i_{k}, n, t_{k}}=$ 0 . And $\rho_{i_{j}, m, t_{j}}$ denotes whether subcarrier $m$ is assigned to user $i_{j} \in \mathcal{A}_{t_{j}}$ in the cell $j$, then $\rho_{i_{j}, m, t_{j}}=1$, otherwise $\rho_{i_{j}, m, t_{j}}=0$. We assume that each particular subcarrier is allocated to more than one user. In addition the number of users which are served by each subcarrier is less than or equal to number of transmitting antennas.

\section{RESOURCE ALLOCATION ALGORITHM}

This problem is mixed-integer and nonconvex and we propose a heuristic method to solve (4). To reduce the computational complexity, first we find the subcarrier assignment and in the second stage, we employ ZF precoder for allocating power to users in the particular subcarrier by using a fixed subcarrier assignment. In the fist stage, the algorithm for subcarrier allocation in the cell $k$ is described according to Gale-Shapley algorithm. Similarly for the cell $j$. As given in the stable marriage problem, we assume that role of men is assumed by the users, while the role of women is assumed by 
the subcarriers. If the number of subcarriers is not enough to serve all of it's users, the base station will ask the remaining users to utilize the shared band. Note that in the cell $j$, we repeat the same process as in the cell $k$. The algorithm for subcarrier allocation with ZF precoder design is described in Table I.

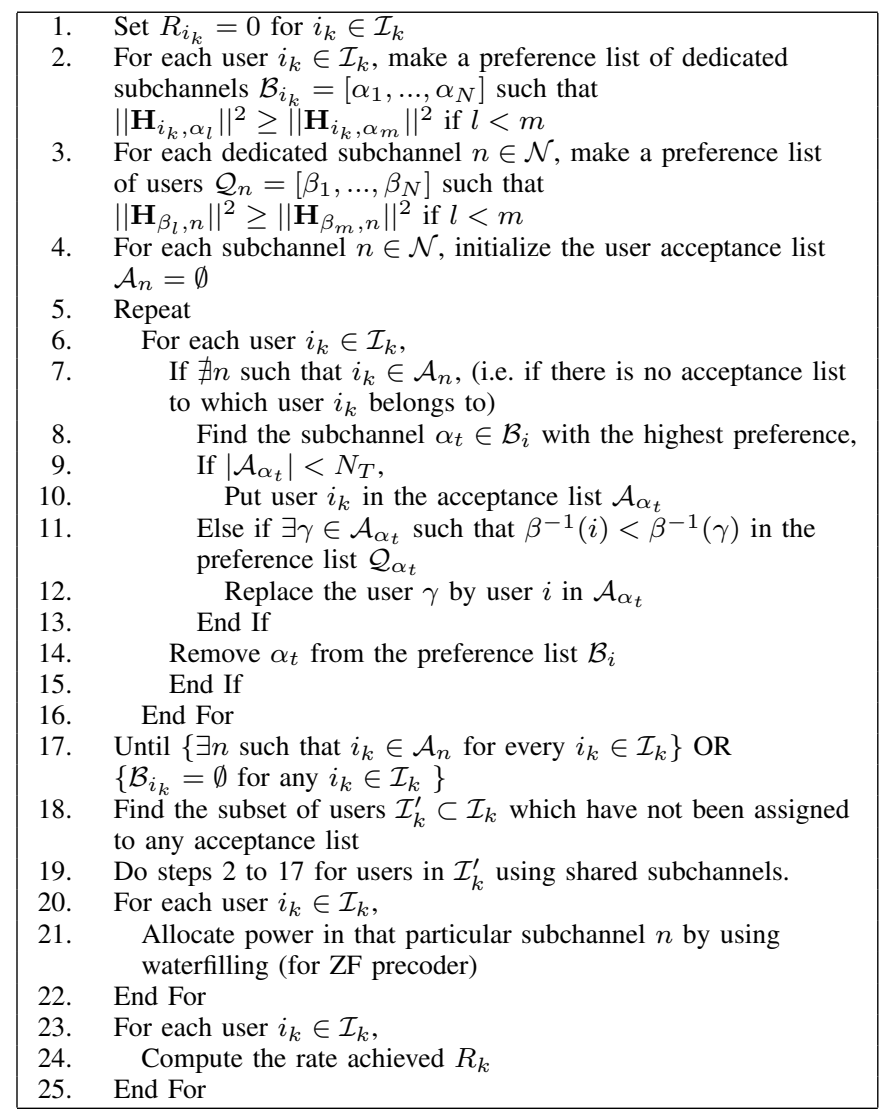

\section{TABLE I}

SubCarRier Allocation by USING GALE-Shapley Algorithm with ZF PRECODER

\section{PRECODER DESIGN}

We consider zero forcing precoder for both base stations.

\section{A. Zero Forcing Precoder}

In this section, we find the user power allocation based on zero forcing (ZF) precoding [10] for a fixed subcarrier assignment. Assume that in the cell $k$, we have chosen $\rho_{i_{k}, n, t_{k}}$ for each subcarrier $n$. And similar to the cell $j$, we have chosen $\rho_{i_{j}, m, t_{j}}$ for each subcarrier $m$. With ZF precoding design, it provides perfect orthogonality across the interfering broadcast signal. Thus, in the shared band, both intracell and intercell interference are eliminated.

For the dedicated band of both cells, the ZF precoder design is to null the intracell interference across other users allocated to the same subcarriers. In the cell $k$, the precoder $\mathbf{T}_{j_{k}, n}$ is designed to achieve zero interference between users i.e. for user $i_{k}$ assigned to utilize the subcarrier $n$ in the dedicated band,

$$
\mathbf{H}_{i_{k}, n} \mathbf{T}_{j_{k}, n}=0, \quad i_{k} \neq j_{k}, \quad i_{k}, j_{k} \in \mathcal{A}_{t_{k}},
$$

similarly in the cell $j$. Thus, ICI is cancelled in both dedicated and shared bands of both cells. In he cell $k$, we define $\mathcal{S}_{n}$ contains the indexes of users $i_{k}, i_{k} \in \mathcal{A}_{t_{k}}$ assigned to subchannel $n$. and let $g_{n}=\left|\mathcal{S}_{n}\right|, \forall n$. The channel vectors of selected uses in the rows of a $g_{n} \times N_{T_{k}}$. is as, $\mathbf{H}_{\mathcal{S}_{n}}=$ $\left[\mathbf{h}_{\mathcal{S}_{n}(1), n}, \ldots, \mathbf{h}_{\mathcal{S}_{n}\left(g_{n}\right), n}\right]^{T}$, where $\mathcal{S}_{n}(b)$ is $b$ th user in the set $\mathcal{S}_{n}$. The beamforming vectors in columns of $N_{T_{k}} \times g_{n}$ matrix for each subchannel $\mathbf{T}_{\mathcal{S}_{n}}=\left[\mathbf{T}_{\mathcal{S}_{n}(1), n}, \ldots, \mathbf{h}_{\mathcal{S}_{n}\left(g_{n}\right), n}\right]$. Then, the $\mathrm{ZF}$ constraints in (5) are rewritten as,

$$
\mathbf{H}_{\mathcal{S}_{n}} \mathbf{T}_{\mathcal{S}_{n}}=\operatorname{diag}\left(\sqrt{\mathbf{p}_{n}}\right), \forall n
$$

where $\mathbf{p}_{n}=\left\{p_{a, n}\right\}$ is users power vector comprised of $p_{a, n}=$ $\mathbf{h}_{\mathcal{S}_{n}(a), n} \mathbf{T}_{\mathcal{S}_{n}(a), n}, a \in\left\{1, \ldots, g_{n}\right\}$.

With the restricted direction of $\mathbf{T}_{\mathcal{S}_{n}}$ to the pseudo-inverse of matrix $\mathbf{H}_{\mathcal{S}_{n}}$ as done in [11], we can obtain (6) as,

$$
\mathbf{T}_{\mathcal{S}_{n}}=\mathbf{H}_{\mathcal{S}_{n}}^{+} \operatorname{diag}\left(\sqrt{\mathbf{p}_{n}}\right), \forall n
$$

By replacing (7) in (4), we can obtain the power constraint of the $\mathrm{BS} k$ as,

$$
\sum_{i_{k}=1}^{I_{k}} \sum_{n=1}^{N} \alpha_{i_{k}, n} p_{i_{k}, n}-P \leq 0
$$

where

$$
\begin{gathered}
\alpha_{i_{k}, n}=\left\{\begin{array}{lr}
{\left[\left(\mathbf{H}_{\mathcal{S}_{n}}^{+}\right)^{H} \mathbf{H}_{\mathcal{S}_{n}}^{+}\right]_{a, a}} & \text { if } i_{k}=S_{n}(a), \\
0 & \text { otherwise }
\end{array}\right. \\
p_{i_{k}, n}=\left\{\begin{array}{lr}
p_{a, n} & \text { if } i_{k}=S_{n}(a), \\
0 & \text { otherwise }
\end{array}\right.
\end{gathered}
$$

Then, we replace $\left(\mathbf{H}_{\mathcal{S}_{n}} \mathbf{T}_{\mathcal{S}_{n}}\right)^{2}$ by $p_{i_{k}, n}$ in the data rate equation of the cell $k$. If the subcarriers in the dedicated band is not enough to serve all users, the remaining users will be assigned to use the shared band. The ZF precoder in the shared band can be written in the similar way as the dedicated band.

In the cell $j$, we replace $\left(\mathbf{H}_{\mathcal{S}_{m}} \mathbf{T}_{\mathcal{S}_{m}}\right)^{2}$ by $p_{i_{j}, m}$ in the same way. By choosing $\rho_{i_{k}, n, t_{k}}$ and $\rho_{i_{j}, m, t_{j}}$ as in subcarrier allocation stage, we do not need subcarrier constraints in power allocation part. The total achievable rate of user $i_{k}$ and user $i_{j}$ which allocated in the particular subcarrier $n$, and $m$ can be reformulated as $R_{i_{k}}=\sum_{n \in \mathcal{N}} \log _{2}\left(1+\frac{p_{i_{k}, n}}{z_{i_{k}, n}}\right)$ and $R_{i_{j}}=\sum_{m \in \mathcal{M}} \log _{2}\left(1+\frac{p_{i_{j}, m}}{z_{i_{j}, m}}\right)$, respectively. The total sum rate optimization problem is rewritten by,

$$
\begin{array}{rc}
\max _{p_{i_{k}, n}, p_{i_{j}, m}} & \sum_{i_{k} \in I_{k}} R_{i_{k}}+\sum_{i_{j} \in I_{j}} R_{i_{j}} \\
\text { subject to } & \sum_{i_{k} \in I_{k}} \sum_{n \in N} \alpha_{i_{k}, n} p_{i_{k}, n} \leq P_{k}^{\max } \\
& \sum_{i_{j} \in I_{j}} \sum_{m \in M} \alpha_{i_{j}, m} p_{i_{j}, m} \leq P_{j}^{\max },
\end{array}
$$

The sum rate maximization problem in (9) becomes convex and can be solved by using Karush-Khun-Tucker (KKT) 
conditions. Thus, it yields to water-filling power allocation. For the cell $k$, we can write Lagrangian dual problem as,

$$
\begin{aligned}
L & =\sum_{i_{k}=1}^{I_{k}} \sum_{n=1}^{N} w_{i_{k}} \log _{2}\left(1+\frac{p_{i_{k}, n}}{z_{i_{k}, n}}\right) \\
& -\lambda\left(\sum_{i_{k}=1}^{I_{k}} \sum_{n=1}^{N} \alpha_{i_{k}, n} p_{i_{k}, n}-P_{k}^{\max }\right)
\end{aligned}
$$

where $\lambda$ is the Langrange multiplier. With the first order derivative of $L$ with respect to $p_{i_{k}, n}$ set equal to zero with complementary slackness, it leads to

$$
p_{i_{k}, n}^{*}=\left(\frac{1}{\lambda^{*} \alpha_{i_{k}, n}}-z_{i_{k}, n}\right)^{+}
$$

where $(.)^{+}=\max (0,$.$) . For the cell j$, we can write KKT conditions in a similar way. Thus, the power allocated to each user $i_{j}$ in the cell $j$ for the particular subcarrier $m$ can be given as,

$$
p_{i_{j}, m}^{*}=\left(\frac{1}{\lambda^{*} \alpha_{i_{j}, m}}-z_{i_{j}, m}\right)^{+}
$$

\section{Numerical Results}

The numerical results illustrate maximizing sum rate throughput for two small cell multiuser system. Rayleigh fading channels are considered for downlink transmission. The transmit beamformers for both cells are employed with zero forcing beamforming and hence they cancel intra-cell and inter-cell interference. The number of antennas at both base stations $\left(N_{T_{k}}\right)$ is set to be 4 and 5 . Each user utilizes a single antenna in both cells. The number of subcarriers for dedicated band $N$ and $M$ for the cell $j$, and $k$ is set to be 1,3 , and 4 . We denote $N_{\text {share }}$ and $M_{\text {share }}$ as number of subcarriers allocated in shared band for cell $k$ and cell $j$, where $j \neq k$, respectively.

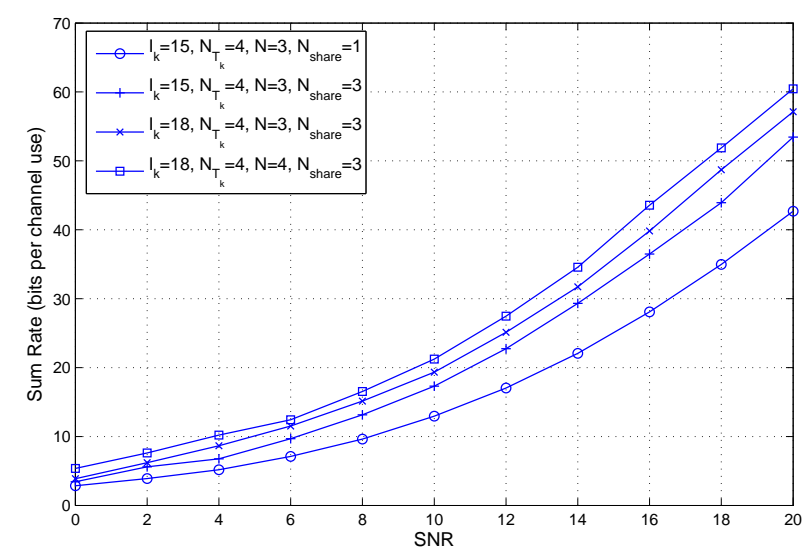

Fig. 2. Sum rate versus SNR for single cell

Fig. 2. demonstrates the sum rate for asingle cell. The number of users $\left(I_{k}\right)$ are 15 and 18 for each case. The number of subcarriers for dedicated band $N$ is 3 , and 4 . The number of subcarriers for shared band is 1 and 3 . We can see that when number of subcarriers in the shared band $\left(N_{\text {share }}\right)$ is higher, the

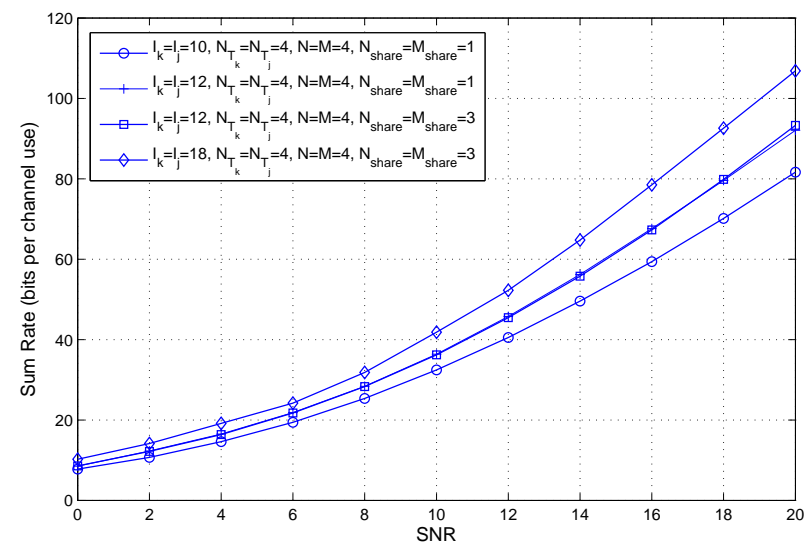

Fig. 3. Sum rate versus SNR for two cells with different number of users and number of subcarriers in the shared band

overall sum rate is improved dramatically. In addition, when $N_{T_{k}}$ and $I_{k}$ increase, the sum rate becomes higher.

Fig. 3. shows the sum rate for two cells. The number of users in the cell $k$ and $j$ is denoted as $I_{k}$ and $I_{j}$, respectively. $I_{k}$ and $I_{j}$ are set to be 10,12 and 18 for each case. The base station $k$, and $j$ utilize 4 antennas. It can be observed that when $I_{k}=I_{j}=12, N_{T_{k}}=N_{T_{j}}=4, N=M=4$, by varying $N_{\text {share }}$ and $M_{\text {share }}$ to be 1 and 3 , the overall sum rate does not change. This is due to the fact that all users can be allocated subcarriers in the dedicated band. Both base stations do not assign users to utilize the shared band. The number of users which can be served in the dedicated band is equal to the number of transmit antennas multiplied by the number of subcarriers.

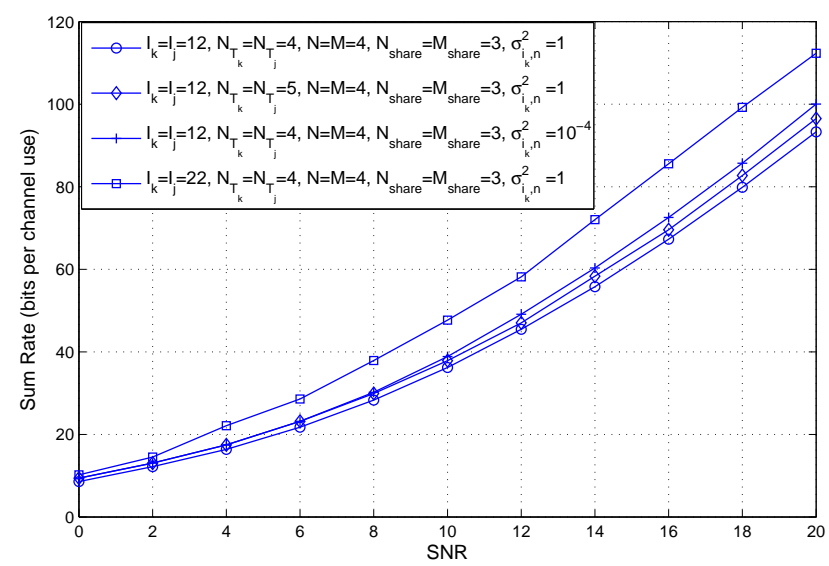

Fig. 4. Sum rate versus SNR for two cells with different number of antennas, number of users and noise variance

Fig.4. illustrates the sum rate of two cells. When the number of transmit antennas $N_{T_{k}}, N_{T_{j}}$ increases, the sum rate becomes higher. Also, if the noise variance is lower such that $\sigma_{i_{k}, n}=$ $10^{-4}$ and $\sigma_{i_{j}, m}=1$, the over all sum rate throughput will increase. Moreover, if the number of users is higher and they 
are allocated to both dedicated and shared bands, the overall sum rate improves significantly.

\section{CONCLUSION}

We proposed co-primary spectrum sharing concept for the MISO small cell network. The sum rate maximization problem is studied with subcarrier and power allocation. The resource allocation problem becomes non-convex and thus we obtain a sub-optimal solution. We seperate the main obtimization into two sub-problems. In the first sub-problem, we allocate the subcarriers to each user by employing Gale-Shapley algorithm. Each subcarrier can be allocated to multiple users. In the second sub-problem, ZF precoders are utilized at both base stations to cancel both intra-cell and inter-cell interference.

Numerical results illustrate the over all sum rate for both base stations with uncorrelated antennas. We also show the overall sum rate throughput with different number of subcarriers served in dedicated and shared bands for both cells. With different number of antennas at the base stations and number of users, the sum rate is explored. We invetigate the scenario when the number of users is higher than the number of transmit antennas multiplied by number of subcarriers in the dedicated band. Then we allocate the remaining users into the dedicated band after finishing user allocation into the dedicated band. The sum rate enhances significantly, especially when the number of subcarriers in the shared band is increased. Moreover, when the number of transmit antennas increases, the sum rate is improved.

\section{REFERENCES}

[1] M. Palola, T. Rautoio, M. Matinmikko, J. Prokkola, M. Mustonen, M. Heikkila, T. Kippola, S. Yrjola, V. Hartikainen, L. Tudose, A. Kivinen, J. Paavola, J. Okkonen, M. Makelainen, T. Hanninen, and H. Kokkinen, "Licensed shared access (lsa) trial demonstration using real lte network," in Proc. Crowncom, 2014.

[2] S. Yrjola and E. Heikkinen, "Active antenna system enhancement for supporting licensed shared access (lsa) concept," in Proc. Crowncom, 2014.

[3] M. Mustonen, M. Matinmikko, M. Palola, S. Yrjola, J. Paavola, A. Kivinen, and J. Engelberg, "Spectrum sharing and energy-efficient power optimization for two-tier femtocell networks," in Proc. Crowncom, 2014.

[4] Y. Teng, Y. Wang, and K. Hornema, "Co-primary spectrum sharing for denser networks in local area," in Proc. Crowncom, 2014.

[5] Y. Xu, L. Wang, C. Fischione, and V. Fodor, "Distributed spectrum leasing via vertical cooperation in spectrum sharing networks," in Proc. Crowncom, 2014.

[6] S. Hailu, A. A. Dowhuszko, and O. Tirkkonen, "Adaptive co-primary shared access between co-located radio access networks," in Proc. Crowncom, 2014.

[7] I. Ahmad, Z. Feng, A. Hammed, P. Zhang, and Y. Zhao, "Spectrum sharing and energy-efficient power optimization for two-tier femtocell networks," in Proc. Crowncom, 2014.

[8] E. Karipidis, D. Gesbert, M. Haardt, E. Ho, Ka-Ming. an Jorswieck, E. G. Larsson, J. Li, J. Lindblom, C. Scheunert, M. Schubert, and N. Vucic, "Transmit beamforming for inter-operator spectrum sharing," in Proc. Future Network and Mobile Summit Conference, 2011.

[9] D. Gale and L. Shapley, "College admissions and stability of marriage," The American Mathematical Monthly Jstor, vol. 69, no. 1, pp. 9-15, Jan 1962.

[10] A. Wiesel, Y. Eldar, and S. Shamai, "Zero-forcing precoding and generalized inverses," IEEE Trans on Signal Processing, vol. 56, no. 9, pp. 4409-4418, Sept 2008.
[11] D. Perea-Vega, A. Girard, and J. Frigon, "Dual-based bounds for resource allocation in zero-forcing beamforming ofdma-sdma systems," EURASIP Journal on Wireless Communications and Networking, Feb. 2013. 